\title{
Comparación pareada como método de evaluación de competencias transversales en materia de sostenibilidad
}

\section{Ignacio J. Navarro ${ }^{\mathrm{a}}$, Víctor Yepes ${ }^{\mathrm{b}}$ y José V. Martic}

${ }^{a}$ Departamento de Ingeniería de la Construcción y Proyectos de Ingeniería Civil. Universitat Politècnica de València. ignamar1@cam.upv.es ${ }^{b}$ ICITECH. Equipo de Innovación y Calidad Educativa EXCELCON. Departamento de Ingeniería de la Construcción y Proyectos de Ingeniería Civil. Universitat Politècnica de València. vyepesp@cst.upv.es y ${ }^{c}$ ICITECH. Equipo de Innovación y Calidad Educativa EXCELCON. Departamento de Ingeniería de la Construcción y Proyectos de Ingeniería Civil. Universitat Politècnica de València. jvmartia@,cst.upv.es

\section{Abstract \\ The evaluation of the transversal competencies acquired throughout university training is a fundamental objective to guarantee the adequate education of students. With the recently established Sustainable Development Goals, there is a growing demand for the acquisition of transversal competencies oriented to this end, and their development and evaluation is becoming more urgent over time. In the context of sustainability, the ability of students to think critically is specially relevant. In view of the lack of consensus on how to determine the degree of acquisition of transversal competencies, an objective procedure is proposed here to evaluate critical thinking based on the resolution of case studies, combined with the application of the Analytical Hierarchy Process for multi-criteria decision making. The application of this process makes it possible to objectively determine the consistency that students implicitly emit in their judgments, offering a valuable tool to evaluate their critical thinking and the clarity with which they perceive sustainability and its implications in the development of their future professional practice.}

Keywords: transversal competencies, critical thinking, Sustainable Development Goals, Analytical Hierarchy Process, consistency, case study.

\section{Resumen}

La evaluación de las competencias transversales adquiridas a lo largo de la formación universitaria es un objetivo fundamental para garantizar la adecuada formación de los alumnos. Con los recientemente establecidos Objetivos de Desarrollo Sostenible, la demanda de competencias transversales orientadas a tal fin es cada vez mayor y, su desarrollo y evaluación, más urgente. En el contexto de la sostenibilidad, resulta particularmente relevante la capacidad de pensamiento crítico de los estudiantes. Ante la falta de consenso en cuanto a cómo determinar el grado de adquisición de las competencias transversales, se propone un procedimiento objetivo orientado a la evaluación del pensamiento crítico basado en la resolución de casos de estudio, combinados con la aplicación del Proceso Analítico Jerárquico de decisión multicriterio. La aplicación de este proceso permite determinar, de forma objetiva, la coherencia implicita de los alumnos en sus juicios y ofrece, por lo tanto, una herramienta valiosa para evaluar su capacidad de pensamiento crítico y la claridad con la que perciben la sostenibilidad y sus implicaciones en el desarrollo de su futura práctica profesional. 
Palabras clave: competencias transversales, pensamiento crítico, Objetivos de Desarrollo Sostenible, Proceso Analítico Jerárquico, consistencia, caso de estudio.

\section{Introducción}

En 2015, las Naciones Unidas aprobaron de forma unánime el documento "Transformando nuestro mundo: la Agenda 2030 para el Desarrollo Sostenible" (Asamblea General de las Naciones Unidas, 2015), un acuerdo que aspira a sentar las bases del desarrollo de una sociedad mundial orientada hacia la consecución de un futuro mejor para el año 2030. Uno de los elementos clave de esta Agenda es el establecimiento de los 17 Objetivos de Desarrollo Sostenible (ODS), prioridades enfocadas a abordar los problemas globales más urgentes: proteger el medio ambiente y la degradación resultante del cambio climático, erradicar el hambre y la pobreza en todas sus formas, fomentar sociedades justas y libres o garantizar un estado de bienestar social y prosperidad, entre otros. Estos ODS, que suponen una continuación de los Objetivos de Desarrollo del Milenio, no son legalmente vinculantes, aunque se espera que tengan un gran impacto sobre las decisiones estratégicas de los gobiernos en los próximos años.

En este contexto, las universidades juegan un papel fundamental para la consecución de los ODS, en la medida en que permiten formar profesionales con capacidad para resolver adecuadamente los complejos desafíos económicos, ambientales y sociales planteados en la Agenda. Las universidades pueden proporcionar el conocimiento, las innovaciones y las soluciones necesarias a través de la investigación y del desarrollo de competencias técnicas específicas a sus alumnos. Sin embargo, para poder formular soluciones adecuadas, es necesario comprender los desafíos correctamente. Para ello es preciso, en el contexto del cumplimiento de los ODS, hacer especial hincapié en el desarrollo de competencias transversales en los planes de estudio universitarios (Kestin et al., 2017). El desarrollo de este tipo de competencias es especialmente relevante para los perfiles de ingeniería y arquitectura. A los ingenieros y arquitectos, además de exigírseles la adquisición de habilidades transversales como el pensamiento crítico, el pensamiento sistémico, la autoconciencia, la responsabilidad social y competencias normativas, se les requiere explícitamente a través del ODS 9 que sean capaces de diseñar infraestructuras sostenibles. Si bien el diseño de infraestructuras sostenibles está siendo el foco de atención de numerosos investigadores en los últimos años (Sierra et al., 2016, Sánchez-Garrido y Yepes, 2020, Martí et al., 2015, Penadés-Plà et al., 2020), el sistema de educación universitaria todavía requiere una importante transformación para garantizar la adquisición de competencias transversales orientadas hacia este fin.

Durante los últimos años, la evaluación del grado de adquisición de dichas competencias transversales se ha convertido en un objetivo estratégico para la Universitat Politècnica de València, impulsado por el Vicerrectorado de Estudios, Calidad y Acreditación. La evaluación de competencias es, sin embargo, una tarea compleja, ya que no existe consenso en cuanto a qué capacidades las componen y, por lo tanto, en cuanto a qué habilidades deben ser evaluadas (Cruz et al., 2019). Por otra parte, tampoco es evidente que el profesorado sea capaz de valorar correctamente ciertas competencias. En este sentido, algunos autores recomiendan contar con los alumnos como evaluadores de sus propias habilidades o de las de sus compañeros, aumentando de esta manera su capacidad de pensamiento crítico, así como fomentando también el aprendizaje autónomo mediante la metacognición y la reflexión (Ljungman y Silén, 2008). Sin embargo, la fiabilidad de dicha evaluación queda supeditada a la objetividad y a la fiabilidad del propio alumno en sus valoraciones (Pond, 2007). 


\section{Objetivos}

La Agenda 2030 plantea la necesidad de formar, desde las universidades, profesionales involucrados con el desarrollo sostenible, capaces de comprender el impacto y las consecuencias que sus decisiones tienen sobre el medio ambiente, la sociedad y la economía. Ante la ausencia de criterios objetivos para la evaluación de las competencias transversales que conducen a estos perfiles impulsores de los ODS, el presente trabajo propone una metodología para evaluar de forma objetiva la capacidad del alumnado para emitir juicios consistentes. Dicha consistencia es considerada como una de las habilidades esenciales necesarias para desarrollar un pensamiento crítico (Kallet, 2014) que, a su vez, es una de las competencias transversales fundamentales que debe enseñar la educación orientada al desarrollo sostenible (Straková y Cimermanová, 2018). La coherencia de pensamiento también es un pilar fundamental para otras competencias transversales consideradas clave para el desarrollo de profesionales alineados con los ODS: toma de decisiones, la capacidad de prever escenarios futuros alternativos, así como la capacidad de identificar las complejas conexiones que existen entre las dimensiones ambiental, social y económica de la sostenibilidad (Cebrián y Junyent, 2015, Lambrechts et al., 2013).

\section{Desarrollo de la innovación}

La innovación planteada consiste en fundamentar la evaluación de las competencias transversales de los alumnos relacionadas con la consecución de los ODS a través de casos de estudio, en los cuales se animará al alumnado a hacer un análisis pareado comparativo de los criterios que intervengan en dichos ejercicios. Mediante la aplicación de un Proceso Analítico Jerárquico (AHP, por sus siglas en inglés), es posible determinar de una forma objetiva la coherencia en sus juicios al respecto y, en consecuencia, la claridad con la que son capaces de afrontar y percibir el problema.

\subsection{Evaluación del pensamiento crítico mediante la comparación pareada}

El Proceso Analítico Jerárquico es una metodología desarrollada por Thomas L. Saaty (Saaty, 1980) y aplicable a problemas complejos de decisión multicriterio. Este proceso se emplea para determinar la importancia relativa que tiene cada uno de los criterios considerados en el problema para la resolución del mismo. Para ello, el proceso requiere que el agente decisor compare, dos a dos, los criterios que se han considerado influyentes en la toma de decisión, indicando cómo de importante considera que es un criterio respecto al otro. Esta comparación de prioridades se hace empleando una escala numérica, la llamada escala fundamental de Saaty (tabla 1), según la cual se hace corresponder un valor semántico a un valor numérico manejable matemáticamente.

Tabla 1. Escala Fundamental de Saaty (Saaty, 1980)

\begin{tabular}{cr}
\hline Valor numérico & Planteamiento verbal de la preferencia \\
\hline 1 & El criterio A es igual de importante que el criterio B \\
3 & El criterio A es ligeramente más importante que el criterio B \\
5 & El criterio A es más importante que el criterio B \\
7 & El criterio A es mucho más importante que el criterio B \\
9 & La mayor importancia del criterio A sobre el B está fuera de toda duda \\
$2,4,6,8$ & Valores intermedios entre los anteriores, cuando es necesario matizar \\
\hline
\end{tabular}


Empleando la escala fundamental, se puede construir una matriz cuadrada $A_{n x n}$ denominada matriz de decisión, en la cual se asigna, para cada elemento $a_{i j}$, el valor numérico que refleja el juicio del decisor en su comparación de los criterios $i$ y $j$. Esta matriz debe ser recíproca, es decir, que si $a_{i j}=x$, entonces $a_{i j}=$ $1 / x$. A partir de esta matriz, el método permite obtener la relevancia de cada criterio como los valores del autovector correspondiente al autovalor mayor de la matriz $\left(\lambda_{\max }\right)$.

Para que los pesos se puedan dar por válidos, el método de Saaty exige que la matriz de decisión sea consistente, es decir, que los juicios que el decisor ha emitido al conformar la matriz de decisión sean coherentes entre sí. Una consecuencia directa de dicha coherencia es que $a_{i j} x a_{j k}=a_{i k} \forall i, j, k$. El método AHP establece un procedimiento para calcular el Índice de Consistencia $C I$ de la matriz de decisión como:

$$
\begin{aligned}
& C I=\frac{\lambda_{\max }-n}{n-1} \\
& C R=\frac{C I}{R I}
\end{aligned}
$$

Donde $n$ número de criterios considerados, y $R I$ indica la consistencia de una matriz cuadrada $n x n$ completamente aleatoria. Los pesos obtenidos serán dados por válidos siempre que el ratio de consistencia $C R$ no supere los valores límite mostrados en la tabla 2 (Saaty, 1980).

Tabla 2. Valores de RI y límites de la proporción de consistencia CR (Saaty, 1980)

\begin{tabular}{ccc}
\hline $\begin{array}{c}\text { Número de } \\
\text { criterios } \boldsymbol{n}\end{array}$ & $\begin{array}{c}\text { Índice aleatorio } \\
\boldsymbol{R} \boldsymbol{I}\end{array}$ & $\begin{array}{c}\text { Ratio de consistencia } \\
\text { límite } \boldsymbol{C R}_{\text {lim }}\end{array}$ \\
\hline 2 & 0 & $0 \%$ \\
3 & 0,58 & $5 \%$ \\
4 & 0,9 & $9 \%$ \\
5 & 1,12 & $10 \%$ \\
6 & 1,24 & $10 \%$ \\
7 & 1,32 & $10 \%$ \\
8 & 1,41 & $10 \%$ \\
9 & 1,45 & $10 \%$ \\
10 & 1,49 & $10 \%$ \\
\hline
\end{tabular}

Si el ratio de consistencia $C R$ se acerca a la unidad, esto quiere decir que los juicios emitidos por el decisor y, por lo tanto, la atribución de valores a los elementos $a_{i j}$, ha sido completamente aleatoria, reflejando un conocimiento nulo del problema a resolver. Por el contrario, un ratio de consistencia bajo indica que el decisor tiene una visión clara del problema a resolver, y refleja en consecuencia que el decisor tiene una capacidad de pensamiento crítico suficientemente desarrollada como para poder comprender la complejidad del problema que se trate y emitir juicios coherentes al respecto. Así pues, es precisamente el ratio $C R$, y no los pesos que finalmente se pudieran obtener tras la aplicación del AHP, el concepto capaz de sintetizar la competencia que el decisor tiene de resolver el problema concreto de que se trata. 


\subsection{Caso de Estudio}

Para poder conocer el grado de adquisición de la capacidad de pensamiento crítico por los alumnos a lo largo de su formación en materia de sostenibilidad, se propone el planteamiento de casos de estudio, en los que se proponga al alumno enfrentarse a un problema real. En este caso, se ha trabajado con alumnos del Máster Universitario en Planificación y Gestión en Ingeniería Civil, impartido en la Universitat Politècnica de València (curso académico 2019/2020). Dado que el perfil del alumno de este máster es el de Ingeniero Civil o Arquitecto, se les plantea un problema directamente relacionado con el cumplimiento del ODS 9: Infraestructuras Sostenibles.

El caso de estudio planteado está basado en el estudio de Navarro et al. (2019), en el cual se formulan y analizan los criterios a tener en cuenta para evaluar la sostenibilidad en el diseño de una infraestructura de transporte. En primer lugar, se describe a los alumnos con claridad en qué consiste el problema a resolver. Éste consiste en tratar de encontrar qué tipo de material escoger para el diseño de un puente de hormigón en ambiente marino, de manera que su respuesta a lo largo del tiempo sea lo más sostenible posible. A continuación, se explica de forma pormenorizada cuáles son los criterios que se quiere considerar en la toma de decisión final y las implicaciones que tiene cada uno de ellos. Finalmente, a través de una encuesta online (figura 1), se pide a los alumnos que comparen de forma pareada la importancia que, de acuerdo con su experiencia, debe tener cada criterio respecto al resto. Además, se pide que el encuestado manifieste de forma explícita la certidumbre con la que ha emitido la comparación de cada criterio respecto al resto. Para ello, se le ofrece la posibilidad de escoger de entre las siguientes cinco opciones de respuesta: Ninguna certidumbre, Poca, Moderada, Bastante o Total.

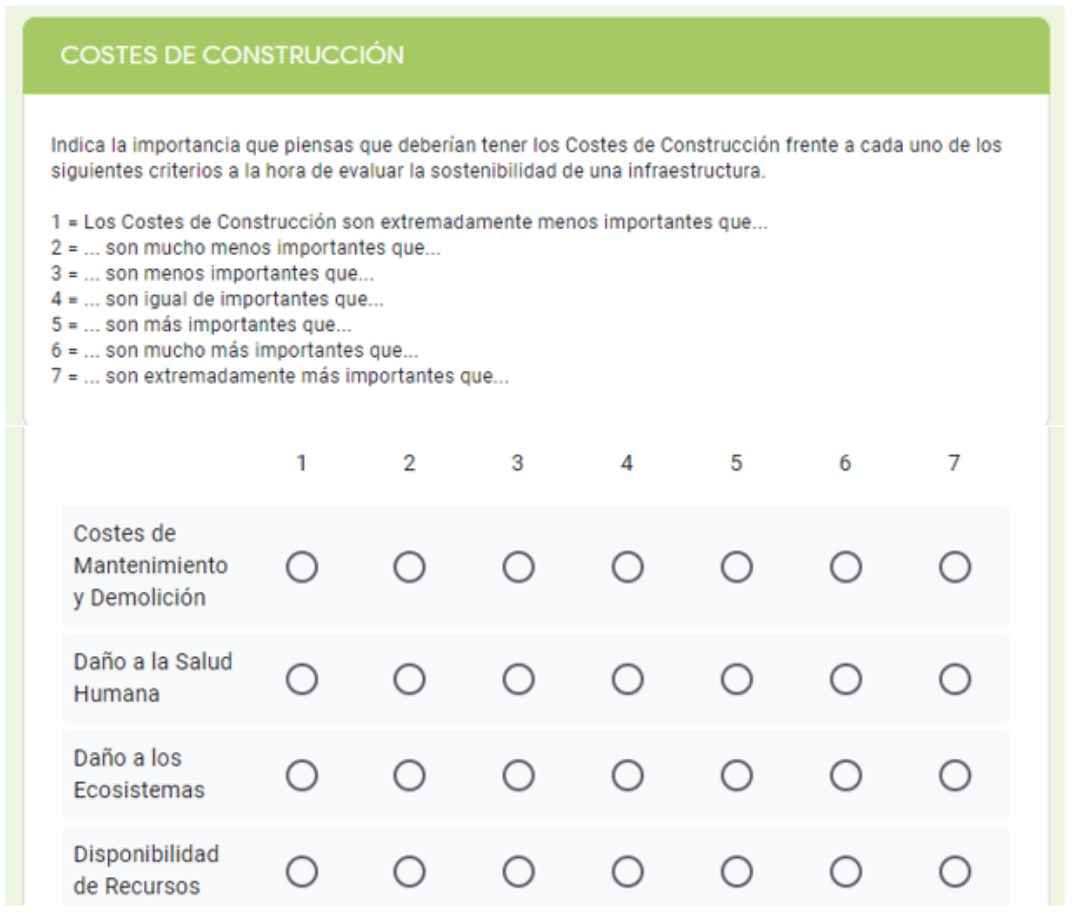

Fig. 1 Extracto de la encuesta

En concreto, la encuesta contempla la comparación de 9 criterios, 2 de ellos económicos, tres ambientales, y 4 referentes a la dimensión social de la sostenibilidad. Cabe comentar que, en la encuesta realizada, no se da al alumno la opción de trabajar directamente con la escala fundamental de Saaty, dada la complejidad del problema. Para el objeto del presente trabajo, basta con trabajar con una escala Likert 
reducida de 7 posibles respuestas, como se observa en la figura 1. A los efectos de poder aplicar la metodología AHP para determinar la consistencia de los alumnos, a los valores de esta escala reducida Likert se les hace coincidir un valor de la escala fundamental de Saaty (tabla 3).

Tabla 3. Correspondencia asumida entre escala Likert de la encuesta y la escala fundamental de Saaty

\begin{tabular}{ccc}
\hline Valor semántico & Valor escala \\
Likert & $\begin{array}{c}\text { Valor escala de } \\
\text { Saaty }\end{array}$ & $1 / 8$ \\
\hline El criterio A es extremadamente menos importante que el B & 1 & $1 / 5$ \\
El criterio A es mucho menos importante que el B & 2 & $1 / 3$ \\
El criterio A es menos importante que el B & 3 & 1 \\
El criterio A es igual de importante que el B & 4 & 3 \\
El criterio A es más importante que el B & 5 & 5 \\
El criterio A es mucho más importante que el B & 6 & 8 \\
\hline
\end{tabular}

En este estudio, la encuesta fue propuesta a un conjunto de 23 alumnos, siendo respondida en su totalidad por todos ellos.

\section{Resultados}

En la tabla 4 se muestran los resultados de la encuesta realizada. En particular, se ha representado el porcentaje de respuestas que, en la muestra de alumnos evaluada, se ha dado a cada posible valor de comparación de la escala Likert propuesta (tabla 3) para cada comparación pareada de criterios. 
Tabla 4. Resultados de la encuesta

\begin{tabular}{|c|c|c|c|c|c|c|c|c|}
\hline Criterio A & Criterio B & 1 & 2 & 3 & 4 & 5 & 6 & 7 \\
\hline \multirow{8}{*}{$\begin{array}{c}\text { Costes de } \\
\text { Construcción } \\
\text { vs... }\end{array}$} & Costes de Mantenimiento & $0 \%$ & $4 \%$ & $4 \%$ & $57 \%$ & $30 \%$ & $4 \%$ & $0 \%$ \\
\hline & Daño a la Salud Humana & $43 \%$ & $26 \%$ & $9 \%$ & $13 \%$ & $0 \%$ & $4 \%$ & $4 \%$ \\
\hline & Daño a los Ecosistemas & $26 \%$ & $17 \%$ & $26 \%$ & $13 \%$ & $13 \%$ & $0 \%$ & $4 \%$ \\
\hline & Disponibilidad de Recursos & $4 \%$ & $17 \%$ & $17 \%$ & $30 \%$ & $26 \%$ & $4 \%$ & $0 \%$ \\
\hline & Generación de Empleo & $0 \%$ & $13 \%$ & $13 \%$ & $35 \%$ & $26 \%$ & $13 \%$ & $0 \%$ \\
\hline & Desarrollo Económico & $4 \%$ & $35 \%$ & $13 \%$ & $13 \%$ & $22 \%$ & $13 \%$ & $0 \%$ \\
\hline & Afección a Usuarios & $9 \%$ & $26 \%$ & $26 \%$ & $13 \%$ & $26 \%$ & $0 \%$ & $0 \%$ \\
\hline & Opinión Pública & $0 \%$ & $13 \%$ & $13 \%$ & $17 \%$ & $30 \%$ & $22 \%$ & $4 \%$ \\
\hline \multirow{7}{*}{$\begin{array}{c}\text { Costes de } \\
\text { Mantenimiento } \\
\text { vs... }\end{array}$} & Daño a la Salud Humana & $48 \%$ & $22 \%$ & $4 \%$ & $13 \%$ & $4 \%$ & $4 \%$ & $4 \%$ \\
\hline & Daño a los Ecosistemas & $22 \%$ & $30 \%$ & $26 \%$ & $0 \%$ & $13 \%$ & $0 \%$ & $9 \%$ \\
\hline & Disponibilidad de Recursos & $4 \%$ & $26 \%$ & $26 \%$ & $26 \%$ & $13 \%$ & $4 \%$ & $0 \%$ \\
\hline & Generación de Empleo & $0 \%$ & $13 \%$ & $30 \%$ & $26 \%$ & $17 \%$ & $9 \%$ & $4 \%$ \\
\hline & Desarrollo Económico & $9 \%$ & $17 \%$ & $22 \%$ & $35 \%$ & $13 \%$ & $4 \%$ & $0 \%$ \\
\hline & Afección a Usuarios & $26 \%$ & $0 \%$ & $35 \%$ & $9 \%$ & $17 \%$ & $0 \%$ & $13 \%$ \\
\hline & Opinión Pública & $0 \%$ & $13 \%$ & $26 \%$ & $13 \%$ & $17 \%$ & $22 \%$ & $9 \%$ \\
\hline \multirow{6}{*}{$\begin{array}{c}\text { Salud Humana } \\
\text { vs... }\end{array}$} & Daño a los Ecosistemas & $0 \%$ & $0 \%$ & $0 \%$ & $48 \%$ & $13 \%$ & $30 \%$ & $9 \%$ \\
\hline & Disponibilidad de Recursos & $13 \%$ & $0 \%$ & $13 \%$ & $9 \%$ & $17 \%$ & $26 \%$ & $22 \%$ \\
\hline & Generación de Empleo & $4 \%$ & $13 \%$ & $4 \%$ & $4 \%$ & $35 \%$ & $17 \%$ & $22 \%$ \\
\hline & Desarrollo Económico & $0 \%$ & $17 \%$ & $0 \%$ & $17 \%$ & $22 \%$ & $26 \%$ & $17 \%$ \\
\hline & Afección a Usuarios & $0 \%$ & $4 \%$ & $13 \%$ & $35 \%$ & $26 \%$ & $13 \%$ & $9 \%$ \\
\hline & Opinión Pública & $13 \%$ & $4 \%$ & $9 \%$ & $0 \%$ & $13 \%$ & $17 \%$ & $43 \%$ \\
\hline \multirow{5}{*}{$\begin{array}{c}\text { Ecosistemas } \\
\text { vs... }\end{array}$} & Disponibilidad de Recursos & $0 \%$ & $0 \%$ & $4 \%$ & $39 \%$ & $39 \%$ & $9 \%$ & $9 \%$ \\
\hline & Generación de Empleo & $0 \%$ & $0 \%$ & $30 \%$ & $13 \%$ & $22 \%$ & $22 \%$ & $13 \%$ \\
\hline & Desarrollo Económico & $0 \%$ & $0 \%$ & $13 \%$ & $39 \%$ & $9 \%$ & $26 \%$ & $13 \%$ \\
\hline & Afección a Usuarios & $0 \%$ & $0 \%$ & $26 \%$ & $26 \%$ & $22 \%$ & $17 \%$ & $9 \%$ \\
\hline & Opinión Pública & $0 \%$ & $4 \%$ & $9 \%$ & $13 \%$ & $17 \%$ & $13 \%$ & $43 \%$ \\
\hline \multirow{4}{*}{ Recursos vs... } & Generación de Empleo & $0 \%$ & $0 \%$ & $22 \%$ & $35 \%$ & $22 \%$ & $13 \%$ & $9 \%$ \\
\hline & Desarrollo Económico & $0 \%$ & $0 \%$ & $26 \%$ & $26 \%$ & $30 \%$ & $13 \%$ & $4 \%$ \\
\hline & Afección a Usuarios & $0 \%$ & $17 \%$ & $30 \%$ & $26 \%$ & $13 \%$ & $4 \%$ & $9 \%$ \\
\hline & Opinión Pública & $9 \%$ & $4 \%$ & $13 \%$ & $22 \%$ & $13 \%$ & $13 \%$ & $26 \%$ \\
\hline \multirow{3}{*}{ Empleo vs... } & Desarrollo Económico & $0 \%$ & $9 \%$ & $13 \%$ & $48 \%$ & $22 \%$ & $9 \%$ & $0 \%$ \\
\hline & Afección a Usuarios & $0 \%$ & $22 \%$ & $9 \%$ & $30 \%$ & $35 \%$ & $4 \%$ & $0 \%$ \\
\hline & Opinión Pública & $9 \%$ & $4 \%$ & $17 \%$ & $26 \%$ & $17 \%$ & $17 \%$ & $9 \%$ \\
\hline $\begin{array}{l}\text { Desarrollo } \\
\text { Económico }\end{array}$ & Afección a Usuarios & $0 \%$ & $4 \%$ & $17 \%$ & $52 \%$ & $13 \%$ & $9 \%$ & $4 \%$ \\
\hline vs... & Opinión Pública & $9 \%$ & $0 \%$ & $4 \%$ & $26 \%$ & $22 \%$ & $22 \%$ & $17 \%$ \\
\hline Usuarios vs... & Opinión Pública & $0 \%$ & $4 \%$ & $9 \%$ & $13 \%$ & $22 \%$ & $35 \%$ & $17 \%$ \\
\hline
\end{tabular}

La tabla 5 muestra la relevancia que, en promedio, y como resultado de esta encuesta, se asigna a cada criterio. Estos valores de relevancias se obtienen a partir de la metodología AHP descrita. Se observa que los alumnos encuestados, como muestra representativa, en este caso, de estudiantes de ingeniería civil y aquitectura recién graduados, consideran mucho más relevante para el diseño sostenible de las infraestructuras los aspectos ambientales en primer lugar, y sociales en segundo, que los criterios 
económicos planteados en el caso de estudio propuesto. Esto refleja la clara voluntad de cambio del tradicional desarrollo enfocado eminentemente hacia la obtención de resultados económicos a un tipo de desarrollo más implicado con el desarrollo de la sociedad y la preservación del medio ambiente.

Tabla 5. Relevancias resultantes de la encuesta

\begin{tabular}{ccc}
\hline Criterio & Tipo de Criterio & Relevancia \\
\hline Costes de Construcción & Económico & $8.29 \%$ \\
Costes de Mantenimiento y Demolición & Económico & $7.98 \%$ \\
Daño a la Salud Humana & Ambiental & $23.26 \%$ \\
Daño a los Ecosistemas & Ambiental & $15.18 \%$ \\
Disponibilidad de Recursos & Ambiental & $9.99 \%$ \\
Generación de Empleo & Social & $8.68 \%$ \\
Desarrollo Económico & Social & $8.84 \%$ \\
Afección a Usuarios & Social & $11.75 \%$ \\
Opinión Pública & Social & $6.04 \%$ \\
\hline
\end{tabular}

En promedio, un $56.5 \%$ de los alumnos encuestados han manifestado un grado de certidumbre moderado a la hora de completar el cuestionario, un $21.7 \%$ ha manifestado bastante certidumbre en sus respuestas, y un $13 \%$ una certidumbre total. El $8.7 \%$ restante de los alumnos ha expresado un bajo grado de certidumbre.

A partir de los resultados de cada encuesta individual se ha podido obtener el ratio de consistencia de cada alumno en sus respuestas (figura 2). Se observa que tan sólo 2 de los 23 han alcanzado una relación de consistenciainferior al 10\% exigido por Saaty para dar por válidos los juicios de una matriz de decisión. Sin embargo, este límite ha sido cuestionado en numerosas ocasiones (Chu y Liu, 2002; Franek y Kresta, 2014; Aguarón et al., 2019) por no tener un fundamento matemático claro. Así, en este trabajo, y a efectos de la evaluación de las competencias transversales en materia de sostenibilidad, vamos a asumir que son consistentes valores de CR de hasta un 15\%. En este caso, se tiene que un 30,4 \% del alumnado ha alcanzado consistencias aceptables en sus juicios. Por otra parte, un $43.5 \%$ de los alumnos han obtenido unos valores de CR por encima del $25 \%$. Estos valores tan elevados indican una falta de coherencia y fiabilidad en sus juicios bastante considerable, reflejando una falta de pensamiento crítico para afrontar un problema, en este caso ingenieril, desde la perspectiva de la sostenibilidad. 


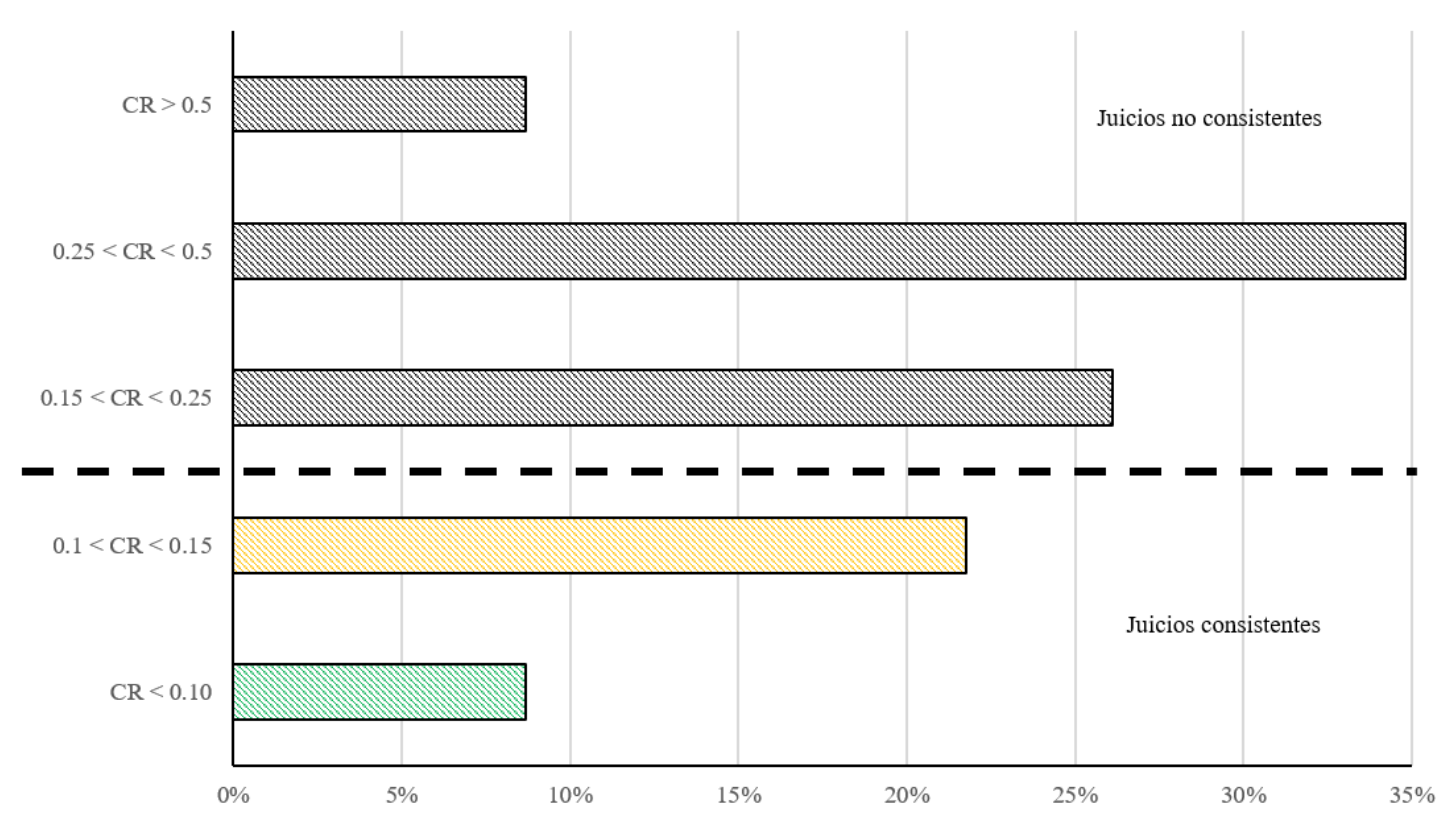

Fig. 2 Ratios de Consistencia (CR) obtenidos

\section{Conclusiones}

La reciente aprobación de la Agenda 2030 plantea la necesidad de que los profesionales formados en un futuro próximo adquieran un conjunto de competencias transversales que les permita orientar su práctica profesional hacia un desarrollo sostenible de la sociedad. El presente trabajo propone una metodología para detectar el grado de desarrollo de la competencia transversal de pensamiento crítico en cualquier fase del aprendizaje de los alumnos. Para ello, se plantea un sistema de evaluación indirecta, basado en el planteamiento de casos de estudio prácticos. Estos casos de estudio, personalizados para cada disciplina universitaria, pretenden motivar al alumnado y fomentar dicho pensamiento crítico y capacidad de análisis, todo ello enfocado desde el punto de vista de la sostenibilidad. La herramienta propuesta permite conocer, a través de la coherencia emitida por los alumnos en sus respuestas de forma implícita, en qué grado el alumno ha desarrollado su capacidad de pensamiento crítico para enfrentarse a problemas de diseño sostenible. El sistema de evaluación propuesto nos ofrece un mapa objetivo de cómo los alumnos perciben el problema del diseño sostenible de las infraestructuras, y permite conocer si es necesario modificar la estrategia docente para salvar las lagunas que se puedan detectar en su formación tras el análisis de los resultados obtenidos.

\section{Referencias}

AGUARÓN, J., ESCOBAR, M. T., MORENO-JIMÉNEZ, J. M. y TURÓN, A. (2019). "AHP-Group Decision Making Based on Consistency" en Mathematics, vol. 7, issue 3, p. 242.

ASAMBLEA GENERAL DE LAS NACIONES UNIDAS (2015). "Transforming our world: The 2030 Agenda for Sustainable Development". A/RES/70/1 (21 October).

CEBRIÁN, G. y JUNYENT, M. (2015) "Competencies in Education for Sustainable Development: Exploring the Student Teachers' Views" en Sustainability, vol. 7, issue 3, p. 2768-2786. 
CHU, P. y LIU, J. K. (2002). "Note on consistency ratio" en Mathematical and Computer Modelling, vol. 35, issues 9-10, p. 1077-1080.

CRUZ, M. L., SAUNDERS-SMITS, G., y GRONE, P. (2019) "Evaluation of competency methods in engineering education: a systematic review" en European Journal of Engineering Education, DOI: 10.1080/03043797.2019.1671810.

FRANEK, J. y KRESTA, A. (2014). “Judgement scales and consistency measure in AHP”. En: Enterprise and the Competitive Environment 2014 conference, 6-7 March 2014, Brno, República Checa.

KALLET, M. (2014).Think smarter: Critical thinking to improve problem-solving and decision-making skills. Nueva Jersey: Wiley.

KESTIN, T., VAN DEN BELT, M., DENBY, L., ROSS, K., THWAITES, J. y HAWKES, M. (2017). “Getting started with the SDGS in universities - A guide for universities, higher education institutions, and the academic sector". Sustainable Development Solutions Network (SDSN) Australia/Pacific.

LAMBRECHTS, W., MULÀ, I., CEULEMANS, K., MOLDEREZ, I., GAEREMYNCK, V. (2013). “The integration of competences for sustainable development inhigher education: an analysis of bachelor programs in management" en Journal of Cleaner Production, vol. 48, p. 65-73.

LJUNGMAN, A. G. y SILÉN, C. (2008). "Examination involving students as peer examiners" en Assessment \& Evaluation in Higher Education, vol. 33, issue 3, p. 289-300.

MARTÍ, J.V., YEPES, V. y GONZÁLEZ-VIDOSA, F. (2015). "Memetic algorithm approach to designing of precastprestressed concrete road bridges with steel fiber-reinforcement" en Journal of Structural Engineering, vol. 141, issue 2, p. 04014114.

NAVARRO, I.J., YEPES, V. y MARTÍ, J.V. (2019). "Sustainability assessment of concrete bridge deck designs in coastal environments using neutrosophic criteria weights" en Structure and Infrastructure Engineering, DOI: 10.1080/15732479.2019.1676791.

PENADÉS-PLÀ, V., GARCÍA-SEGURA, T. y YEPES, V. (2020). "Robust design optimization for low-cost concrete box-girder bridge" en Mathematics, vol. 8, p. 398.

POND, K. (2007). "Student Experiences of Peer Review Marking of Team Projects" en International Journal of Management Education, vol. 6, issue 1, p. 30-43.

SAATY, T. (1980). The Analytic Hierarchy Process. Nueva York: McGraw-Hill.

SÁNCHEZ-GARRIDO, A.J. y YEPES, V. (2020). "Multi-criteria assessment of alternative sustainable structures for a self-promoted, single-family home" en Journal of Cleaner Production, vol. 258, p. 120556.

SIERRA, L.A., PELLICER, E. y YEPES, V. (2016). "Social sustainability in the life cycle of Chilean public infrastructure" en Journal of Construction Engineering and Management, vol. 142, issue 5, p. 05015020.

STRAKOVÁ,Z. y CIMERMANOVÁ, I. (2018). "Critical thinking development - A necessary step in Higher Education transformation towards sustainability” en Sustainability, vol. 10, issue 10, p. 3366. 